\title{
Misdiagnosis of pulmonary tuberculosis and associated factors in peripheral laboratories: a retrospective study, Addis Ababa, Ethiopia
}

\author{
Daniel Melese Desalegn ${ }^{1,2^{*}}$, Kumera Terfa Kitila ${ }^{1,2}$, Hanna Mekonnen Balcha' , Chalachew Sisay Gebeyehu', \\ Yohannes W. Kidan' ${ }^{1}$ Kassayenew Amare', Daniel Dejene', Merone Seifu', Addis Zewdie', Abiyot Tenna', \\ Tinsae Kidanemariam Hailu' ${ }^{1}$ Boja Dufera Taddese ${ }^{1}$ and Abrham Tesfaye Bika ${ }^{1}$
}

\begin{abstract}
Objective: Sputum smear microscopy reading errors are likely to result in failure to detect persons with infectious TB. This study was intended to review misdiagnosis of pulmonary TB and associated factors in peripheral laboratories.

Results: During the study period 1033 (10.5\%) sputum smear positive and 8783 (89.5\%) smear negative slides were reported by peripheral laboratories. The slides were re-read by the central referral laboratories (CRLs) as the reference standard reading. Of 1033 positive slides reported by peripheral laboratories, 25 (2.4\%) were false positive. Out of 8783 smear negative slides reported by peripheral laboratories, 35 (0.4\%) were false negative. The sensitivity, specificity, positive predictive value and negative predictive value of peripheral laboratories were 96.64, 99.72, 97.58, and 99.61\% respectively. The peripheral laboratories and CRLs have an observed agreement (Po) of 0.9939. Of 135 peripheral laboratories, 93 (68.9\%) read negative and positive slides correctly, 49 (36.3\%) did not have lens cleaning tissue papers, 11 (8.1\%) lacked frosted slides, and 14 (10.4\%) had shortage of reagents. As conclusions, the peripheral laboratories and CRLs had high agreement for sputum smear microscopy reading. However, a few TB cases were misdiagnosed despite having the disease; these individuals might continue to spread the infection in the community.
\end{abstract}

Keywords: External quality assessment, ZN sputum smear microscopy, Addis Ababa

\section{Introduction}

Laboratory services continue playing critical role in diagnosing TB and treatment monitoring $[1,2]$. However, it requires strong quality assurance (QA) systems be in place [3-6]. In contrary, reading errors in ZN-stained sputum smear microscopy are likely to result in failure to detect persons with infectious $\mathrm{TB}$, consequently continues to spread infection in the community, or may unnecessary treated for non TB cases [5-7]. Moreover,

\footnotetext{
*Correspondence: danimelese2013@gmail.com

${ }^{1}$ Addis Ababa Public Health Research and Emergency Management Core Process, Addis Ababa City Administration Health Bureau, Addis Ababa, Ethiopia

Full list of author information is available at the end of the article
}

in adequately training, erratic reagent, supplies, and poor equipment maintenance inflate the problems.

Accurate and reliable TB microscopy result relies on external quality assessment (EQA) programs that support, train, and monitor testing performance of individual laboratories [3, 8]. External quality assessment comprises proficiency testing, blind-rechecking of samples and on-site evaluation [7-9]. In study setting, blindrechecking programs is crucial EQA method in assessing sputum smear microscopy reading performance of the laboratory $[7,8]$. It provides an opportunity to assess quality performance elements including specimen quality, smear size and thickness, and quality of staining. All these information may be very useful to assessing possible reasons for false positive or false negative results, and 
implementing plans for retraining and corrective action $[3,9,10]$.

Information concerning the performance of EQA in private and public laboratories in Addis Ababa is very limited; hence we reviewed 135 public and private health facilities' ZN sputum smear microscopy EQA performance, to determine misdiagnosis of pulmonary $T B$ cases and associated factors in private and public health facilities in Addis Ababa, Ethiopia.

\section{Main text Methods}

Retrospective review of EQA records in 135 public and private laboratories in Addis Ababa Ethiopia was conducted, from October, 2014 to March, 2016. These peripheral laboratories provide TB diagnostic services and they participate in ZN smear microscopy Regional External Quality Assurance Scheme (REQAS). This study compared the results of peripheral laboratories' ZNstained sputum smear microscopy reading performances with CRLs as a gold standard.

\section{Study subjects}

One hundred thirty five peripheral laboratories (45 privates and 90 public) providing TB diagnostic service to patients in Addis Ababa were considered for the current study.

\section{Data sources and sampling}

Data were collected from 135 health facilities in Addis Ababa. The health facilities' ZN-stained sputum smear microscopy EQA performances and onsite EQA records were used as data source.

\section{Inclusion and exclusion criteria}

All peripheral laboratories having complete EQA performance records in the CRLs data storage, from the years 2014-2016 were included. However, TB laboratory results performed by other than $\mathrm{ZN}$ technique were excluded.

\section{Data management and statistical analysis}

The collected data were checked for completeness and consistency and entered into using Epi-info software. The data were coded and analysed using SPSS version 20.0 (SPSS Inc. Chicago, USA) software. ZN-stained sputum smear microscopy reading agreement between the peripheral microscopy centers and the CRLs were measured by using Kappa (K). Inter-observer variability was assessed on the basis of $\mathrm{k}$-values, with kappa coefficient: $0.81-1=$ almost perfect, $0.61-0.8=$ substantial, $0.41-0.60=$ moderate, $0.21-0.40=$ fair, $0.01-0.20=$ slight and $\leq 0=$ poor agreement between slide readers [11].
Sensitivity, specificity, PPV and NPV of the peripheral laboratories were calculated against the final readings of the CRLs as a gold standard. Bivariate and Multivariate logistic regression model using odds ratio (OR) at 95\% confidence interval $(\mathrm{CI})$ were calculated. $\mathrm{P}$ value less than 0.05 were taken as statistically significant.

\section{Data quality assurances}

Before extracting data from records, data collectors were adequately trained and the collected data were checked for completeness by data collectors and authors. Data collection process was supervised and monitored by the principal investigator.

\section{Operational definition}

Central referral laboratories (CRLs): Regional and intermediate level (Hospital) laboratories serving as ZN smear microscopy a quality assurance (REQAS) centers for peripheral laboratories.

Peripheral laboratories: Laboratories located at a Health Center or private Hospital and different level of Clinics, providing ZN smear microscopy diagnostic services.

Discordant slides: Positive slides read as negative or vice versa.

\section{Result}

ZN-stained sputum smears microscopy reading agreement During the study period 135 peripheral laboratories were involved in REQAS. Among these, 45 (33.3\%) were private and the remaining $90(66.7 \%)$ public health facilities. Of 135 peripheral laboratories, 93 (68.9\%) correctly read negative and positive sputum smear slides. The remaining 42 (31.1\%) laboratories misread at least one positive sputum smear slides as negative or vice versa. Out of 42 health peripheral laboratories reported false reading results, 23 (54.8\%) were public and the remaining 19 (45.2\%) were private' laboratories. However, there was no statistically significant difference in sputum smear microscopy false reading results between public and private laboratories ( $\mathrm{P}$ value $>0.05$ )

Of $9816 \mathrm{ZN}$-stained sputum smear slides were collected from the peripheral laboratories for re-checking, $1033(10.5 \%)$ were reported as positive, and the reaming 8783 (89.5\%) were reported as negative by peripheral laboratories. The slides were re-read by the CRLs, which were considered as the reference standard reading. Of 1033 sputum smear slides reported as positive by peripheral laboratories, 1008 (97.6\%) were true positive, the remaining 25 (2.4\%) were false positive. Out of $8783 \mathrm{spu}-$ tum smear slides reported as negative by peripheral laboratories, 8748 (99.6\%) were true negative, the remaining $35(0.4 \%)$ were false negative. The overall discordant rate 
between peripheral laboratories and CRLs was 60 (0.61\%) (Table 1).

The study has shown an observed agreement (Po) of 0.9939 , expected agreement $(\mathrm{Pe})$ of 0.8109 and calculated kappa value was 0.97 , which indicated almost perfect agreement. The sensitivity, specificity, PPV and NPV of peripheral laboratories in $\mathrm{ZN}$-stained sputum reading was $96.64,99.72,97.58$ and $99.6 \%$ respectively.

\section{Smear quality}

None of the peripheral laboratories assessed met sputum smear quality standards. Of 9816 collected sputum smear slides from the peripheral laboratories, 3475 (35.4\%) specimen quality, 3316 (33.8\%) evenness, 3004 (30.6\%) smear size and 2817 (28.7\%) labeling were found as poor. Moreover, 3846 (39.2\%) smears were prepared with inappropriate thickness, and 3029 (30.9\%) smears were unacceptable staining background cleanness. Compared to the performance of public and private laboratories for each of the quality indices, of 3168 sputum smear slides collected from the private laboratories, 1163 (36.7\%) specimen quality, 1042 (32.9\%) evenness, 991 (31.3\%) smear size, 916 (28.9\%) labeling, 1291 (40.8\%) thickness, and 1039 (32.8\%) staining background were unacceptable. Of 6648 slides collected from the public laboratories, $2312(34.8 \%)$ specimen quality, 2274 (34.2\%) evenness, 2013 (30.3\%) smear size and 1901 (28.6\%) labeling, 2552 (38.4\%) thickness, and 2053 (30.9\%) staining background were found as poor.

\section{Tuberculosis laboratory facilities and infrastructures}

On-site evaluation of EQA was conducted in 135 peripheral laboratories to assess the status of TB laboratory infrastructures and factors affecting the quality of $\mathrm{ZN}$-stained sputum microscopy diagnosis. The entire assessed peripheral laboratories had functional light microscope, 104 (77.0\%) had separate work station for smearing, staining, microscopy examination and recording. Of the total TB laboratories assessed, 79 (58.5\%) had clean, separate and ventilated TB laboratory room, 127

Table 1 Sputum smears microscopy reading agreement between peripheral laboratories and central referral laboratories in Addis Ababa, Ethiopia

\begin{tabular}{lccc}
\hline \begin{tabular}{l} 
Peripheral laboratories smear \begin{tabular}{l} 
results (test value) \\
\cline { 2 - 3 }
\end{tabular} \\
\cline { 2 - 3 }
\end{tabular} & $\begin{array}{l}\text { Central referral laboratories } \\
\text { results (as true value) }\end{array}$ & Total \\
\cline { 2 - 3 } & Positive & Negative & \\
\hline Positive & 1008 & 25 & 1033 \\
Negative & 35 & 8748 & 8783 \\
Total & 1043 & 8773 & 9816 \\
\hline
\end{tabular}

(94.1\%) had regular water supply and 131 (97.0\%) had back up electric power supply (Table 2).

Shortage of supplies including all type of reagents (Carbol-fuchsin, methylene blue and Acid alcohol), lens tissue; frosted slides, internal quality control (IQC) reagents were identified in most peripheral laboratories. Of 135 peripheral laboratories, 49 (36.3\%) did not have lens cleaning tissue papers, 11 (8.1\%) lacked frosted slides, 14 (10.4\%) had shortage of $\mathrm{ZN}$-staining reagents, and nearer to half $(42.2 \%)$ did not filter $1 \%$ carbol-fuchsin during staining of sputum smears. However, all peripheral laboratories used the appropriate personal protective equipment (PPE) when performing TB laboratory procedures, and they used incinerator to dispose sputum, and other sputum contaminated solid wastes (Table 2).

\section{Quality assurance}

There were gaps in having valid internal quality control materials in all assessed laboratories. Among these, 38 (28.1\%) laboratories prepare smears from known positive and negative sputum as internal quality control to check reagents quality before staining patient sample. About 59 (43.7\%) did not check sputum quality before processing. Among 135 visited laboratories, 109 (80.7\%) had standard TB laboratory request form and result log book, 107 (79.3\%) had TB national guideline; manual, SOPs and other reference materials for smear microscopy technique. More than half (76.3\%) of the peripheral laboratories' staffs members were trained on sputum smear microscopy (Table 2). From those who took training, 73 (70.9\%) were from public health facilities.

\section{Factors affecting sputum smear microscopic reading}

In binary logistic regression, false reading of sputum smear microscopy had significant association with checking the quality of sputum before smear preparation, having separate TB laboratory room and filtering carbolfuchsin before staining sputum smear $(\mathrm{P}$ value $<0.05$ ) However, in multivariate logistic regression analysis, false reading had no significant association with these factors ( $P$ value $>0.05$ ) (Table 3$)$.

\section{Discussion}

In the current study, sputum smear microscopy reading agreements between the peripheral laboratories with the CRLs were evaluated, using CRLs as reference standard. The sensitivity, specificity, PPV and NPV of the peripheral laboratories were 96.64, 99.72, 97.58 and 99.61\%, respectively, which is comparable to $95,99.7,93.3$ and 99.7\% respectively reported in Ethiopia and 91.3, 98.9, 92.2 and $98.8 \%$ respectively reported in Argentine [12, 13]. In contrast, it is higher than others studies [14-16]. In present study overall sputum smear reading agreement 
Table 2 TB laboratory diagnostic facilities and infrastructure assessment, in Addis Ababa, Ethiopia

\begin{tabular}{l} 
Variables \\
\hline Separate TB laboratory room \\
Yes \\
No \\
Separate area for TB laboratory works \\
Yes \\
No \\
Regular electric power supply \\
Yes \\
No \\
Regular water supply \\
Yes \\
No
\end{tabular}

Runn IQC slides parallel with routine tasks

$$
\text { Yes }
$$

No

SOPs, guideline and manuals

Yes

No

Functional light microscopes

Yes

No

ZN-staining reagents

Yes

$$
\text { No }
$$

Lens cleaning tissue paper

$$
\text { Yes }
$$

No

IQC reagents

Yes

No

Good storage condition for reagents

$$
\text { Yes }
$$

No

Checking quality of sputum

$$
\text { Yes }
$$

No

Always carbol-fuchsin filtered before use

$$
\text { Yes }
$$$$
\text { No }
$$

Having news slides (frosted slide)

$$
\text { Yes }
$$$$
\text { No }
$$

Re-used sputum container

$$
\text { Yes }
$$

No

Having personal protective equipment

$$
\text { Yes }
$$$$
\text { No }
$$

Number Percent
58.5

41.5

77.0

23.0

97

14.1

94.1

5.9

28.1

71.9

79.3

100

89.6

10.4

36.3

63.7

19.3
Table 2 (continued)

\begin{tabular}{lrc}
\hline Variables & Number & Percent \\
\hline Having functioning incinerator & 135 & 100 \\
Yes & 0 & 0.0 \\
No & 103 & 76.3 \\
Staff received training on ZN sputum smears microscopy & \\
Yes & 32 & 23.7 \\
No & & \\
Having standard TB result registration log-book & 109 & 80.7 \\
Yes & 26 & 19.3 \\
No & & \\
Received EQA feedback & 124 & 91.9 \\
Yes & 11 & 8.1 \\
No & & \\
\hline
\end{tabular}

between the peripheral diagnostic laboratories and CRLs was $99.4 \%$, which is comparable to $99.5 \%$ reported in west Amhara Region [17] and 99.7\%, reported Lima, Peru [18]. However, it is higher compared to $96.8 \%$ reported in south Ethiopia [19] and 87\% reported in Addis Ababa [16] and 89.2\% report in Tanzania [20]. Although, the proportion of sputum smear microscopy reading agreement of $99.4 \%$ achieved is higher than acceptable performance of $>80 \%[7,10,21]$, still disagreement of $0.6 \%$ may indicate a need to improve the quality of $\mathrm{TB}$ microscopic reading.

In this study overall discordant (false reading) results of sputum microscopy reported by the peripheral laboratories was $60(0.6 \%)$, which is lower than the previous reports of $7.8 \%$ in Argentine, 3.5\% in west Amhara Region, 3.2\% in southern Ethiopia, 3.3\% in India, and $5.5 \%$ in eastern part of Ethiopia [13, 17, 19, 22, 23]. It is higher than study reported $0.2 \%$ in Ethiopia and $0.3 \%$ in Taiwan $[12,24]$. These variations might be due to sputum smear microscopy reading is highly dependent on the training, diligence of microscopist and laboratory supplies. The other possible reason, unfiltered fuchsin crystals reagents, weak decoloration of AFB might affect the quality of ZN smear microscopy reading and lead to false reading results $[16,20,26]$.

In the present study specimen quality, evenness, smears size, inappropriate labeling, thickness, and smears with unacceptable staining background cleanness were $35.4,33.8,30.6,28.7,39.2$ and $31.5 \%$ respectively, which is relatively lower than other studies [24-26]. Specimen container, sample transport condition, specimen quality, labeling, smearing, and staining technique including failure to filter carbol fuchsin are some of the factors that compromise $\mathrm{ZN}$ sputum smears quality performance indices [20, 26]. 
Table 3 Factors affecting for ZN sputum smears microscopy reading in peripheral diagnostic facilities, Addis Ababa, Ethiopia

\begin{tabular}{|c|c|c|c|c|c|c|}
\hline \multirow[t]{2}{*}{ Variables } & \multicolumn{2}{|c|}{ False reading } & \multirow[t]{2}{*}{ COR $(95 \% \mathrm{Cl})$} & \multirow[t]{2}{*}{ P value } & \multirow[t]{2}{*}{ AOR $(95 \% \mathrm{Cl})$} & \multirow[t]{2}{*}{$P$ value } \\
\hline & No & Yes & & & & \\
\hline \multicolumn{7}{|c|}{ Separate TB laboratory room } \\
\hline Yes & 31 & 29 & 1 & & & \\
\hline No & 25 & 50 & $2.14(1.06,4.30)$ & $0.033^{*}$ & $0.46(0.74,2.89)$ & 0.41 \\
\hline \multicolumn{7}{|c|}{ Training on ZN microscopy } \\
\hline Yes & 14 & 46 & 1 & & & \\
\hline No & 18 & 57 & $0.96(0.43,2.14)$ & 0.93 & & \\
\hline \multicolumn{7}{|c|}{ Cheek quality of sputum } \\
\hline Yes & 32 & 28 & 1 & & & \\
\hline No & 27 & 48 & $2.03(1.02,4.06)$ & $0.04^{*}$ & $0.63(0.12,3.28)$ & 0.59 \\
\hline \multicolumn{7}{|l|}{ Use new slides } \\
\hline Yes & 6 & 54 & 1 & & & \\
\hline No & 5 & 70 & $1.56(0.45,5.37)$ & 0.49 & & \\
\hline \multicolumn{7}{|c|}{ Filter reagent before use } \\
\hline Yes & 31 & 29 & 1 & & & \\
\hline No & 26 & 49 & $2.02(1.02,4.04)$ & $0.048^{*}$ & $1.54(0.14,4.17)$ & 0.73 \\
\hline \multicolumn{7}{|c|}{ Prepared and use IQC } \\
\hline Yes & 25 & 10 & 1 & & & \\
\hline No & 84 & 16 & $0.47(0.32,1.79)$ & 0.53 & & \\
\hline \multicolumn{7}{|c|}{ Having lenses tissue } \\
\hline Yes & 20 & 15 & 1 & & & \\
\hline No & 66 & 34 & $0.68(0.29,1.17)$ & 0.13 & & \\
\hline \multicolumn{7}{|c|}{ Good reagent storage condition } \\
\hline Yes & 34 & 66 & 1 & & & \\
\hline No & 19 & 16 & $0.43(0.28,1.15)$ & 0.12 & & \\
\hline \multicolumn{7}{|c|}{ Received feed back } \\
\hline Yes & 6 & 69 & 1 & & & \\
\hline No & 5 & 55 & $0.96(0.28,3.30)$ & 0.94 & & \\
\hline \multicolumn{7}{|l|}{ Owner ship } \\
\hline Private & 26 & 19 & 1 & & & \\
\hline Government & 67 & 23 & $0.87(0.42,1.80)$ & 0.47 & & \\
\hline \multicolumn{7}{|c|}{ Having ZN reagents } \\
\hline Yes & 57 & 4 & 1 & & & \\
\hline No & 63 & 11 & $2.49(0.87,12.29)$ & 0.08 & & \\
\hline
\end{tabular}

* Statistically significant $\mathrm{P}<0.05$

In the current study 79 (58.5\%) of the peripheral laboratories having clean, separate and ventilated TB laboratory room, which is higher than $18.2-42 \%$ previously reported in other studies $[15,16,27,28]$. Unventilated TB laboratory room can create unfavorable working environment, which might affect the overall quality of sputum smear microscopy services from pre-analytical to post analytical phases. In addition, this may create a chance of cross contamination [2, 29].

Regarding TB laboratory safety issue, all the peripheral laboratories used the appropriate PPE and disposed sputum and other sputum contaminated solid wastes appropriately. This finding was higher when compared with study conducted in Malawi, which reported most hospitals' laboratories didn't wear white coat, face mark, protective apron and soap for washing hand [27]. The difference might be due to availability of PPE, professionals' attitude towards PPE. A poor laboratory safety practice does not only put the laboratory workers at risk of infection, but also the patients and any other person accessing the laboratory services. 


\section{Conclusion}

In the present study peripheral laboratories and CRLs had high agreement for ZN sputum smear microscopy reading. However, a few TB cases were misdiagnosed despite having the disease; these individuals might continue to spread the infection in the community. This indicates the needs to improve $\mathrm{ZN}$-stain sputum smear microscopy reading to attain the end TB strategy.

\section{Limitations of the study}

- Results of this study depend only on the TB-EQA record review. Therefore, not illustrate providers view.

\begin{abstract}
Abbreviations
AFB: acid fast bacillus (or bacilli); AOR: adjusted odds ratio; Cl: confidence interval; CRLs: central referral laboratories; EQA: external quality assessment; NPV: negative predictive value; COR: crude odds ratio; SPSS: Statistical Package for Social Sciences; PPV: positive predictive value; TB: tuberculosis; WHO: World Health Organization; ZN: Ziehl-Neelsen stain; REQAS: Regional External Quality Assurance Scheme.
\end{abstract}

\section{Authors' contributions}

DMD designed the study, develops the proposal, lead data collection, analysis and writing up the result. BD, and AT critically review and made progressive suggestions in the study. KT, HM, CS, YWK, KA, DD, MS, AZ, AT, TKH were participated in data collection, analysis, interpretation, and write up the result. All authors read and approved the final manuscript.

\section{Author details}

${ }^{1}$ Addis Ababa Public Health Research and Emergency Management Core Process, Addis Ababa City Administration Health Bureau, Addis Ababa, Ethiopia.

${ }^{2}$ Ethiopian Public Health Institute (EPHI), Addis Ababa, Ethiopia.

\section{Acknowledgements}

The authors would like to acknowledge Addis Ababa City Administration health Bureau Pubic Health research and Emergency management core processes. We sincerely thank respective health facilities for their cooperation. Our thanks also extend to all of the CRLs laboratory staffs for their commitment during slides rechecking.

\section{Competing interests}

The authors declare that they have no competing interests.

\section{Availability of data and materials}

All data were presented in the result parts of the manuscript.

\section{Consent for publish}

Not applicable.

\section{Ethical approval and consent to participate}

Ethical approval was obtained from Ethics committee of Addis Ababa City Administration Health Bureau Public Health Research and Emergency management core processes.

\section{Funding}

The authors had no funding support or funding to report.

\section{Publisher's Note}

Springer Nature remains neutral with regard to jurisdictional claims in published maps and institutional affiliations.
Received: 4 July 2017 Accepted: 7 May 2018

Published online: 11 May 2018

\section{References}

1. Onyebujoh PC, Thirumala AK, Ndihokubwayo J-B. Integrating laboratory networks, surveillance systems and public health institutes in Africa. Afr J Lab Med. 2016;5(3):431. https://doi.org/10.4102/ajlm.v5i3.431.

2. World Health Organization, Stop TB Partnership Retooling Task Force, Stop TB Partnership New Diagnostics Working Group. New laboratory diagnostic tools for tuberculosis control;2009. http://www.stopt b.org/assets/documents/global/retooling/Retooling_Stakeholders.pdf. Accessed 22 June 2017.

3. External Quality Assessment for AFB Smear Microscopy. https://www. aphl.org/aboutAPHL/publications/Documents/External_Quality_Asses sment_for_AFB_Smear_Microscopy.pdf. Accessed 4 July 2017.

4. Steingart KR, Ramsay A, Pai M. Optimizing sputum smear microscopy for the diagnosis of pulmonary tuberculosis. Expert Rev Anti Infect Ther. 2007:5(3):327-31.

5. Van Denu A, Portaels F. Limitations and requirements for quality control of sputum smear microscopyfor acid-fast bacilli. Int J Tuberc Lung Dis. 1998;2(9):756-65.

6. World Health Organization, Quality Assurance of Sputum Microscopy in DOTS Programmes. Regional guidelines for countries in the Western Pacific Regional Office for the Western Pacific. Manila:WHO; 2003. http:// www.who.int/ihr/training/laboratory_quality/11_cd_rom_tb_eqa_wpro. pdf. Accessed 30 June 2017.

7. Federal Ministry of Health (FMOH). Guidelines for quality assurance of smear microscopy for tuberculosis diagnosis, vol. 1. Addis Ababa: Federal Ministry of Health (FMOH); 2009. p. 23-30.

8. World Health Organization. Overview of external quality assessment (EQA): module 10, content sheet 10-1. Geneva: World Health Organization; 2011. http://www.who.int/ihr/training/laboratory_quality/10_b_ eqa_contents. Accessed 30 June 2017.

9. Quality Assurance of Sputum Microscopy in DOTS Programmes. Regional guidelines for countries in the Western Pacific. Geneva: World health organization. ISBN: 929061056 5. http://www.who.int/ihr/training/labor atory_quality/11_cd_rom_tb_eqa_wpro.pdf. Accessed 2 June 2017.

10. Laboratory National Tuberculosis Reference. Standard manual for laboratory technicians on sputum smear microscopy. 2nd ed. Bhutan: National Tuberculosis Reference Laboratory; 2011 (Google Scholar).

11. Viera AJ, Garrett JM. Understanding inter-observer agreement: the Kappa Statistic. Fam Med. 2005;37(5):360-3.

12. Melese M, Jerene D, Alem G, Seid J, Belachew F, Kassie Y, et al. Decentralization of acid fast bacilli (AFB) external quality assurance using blind rechecking for sputum smear microscopy in Ethiopia. PLoS ONE. 2016;11(3):e0151366. https://doi.org/10.1371/journal.pone.0151366.

13. Kusznierz GF, Latini OA, Sequeira MD. Quality assessment of smear microscopy for acid-fast bacilli in the Argentine tuberculosis laboratory network, 1983-2001. Int J Tuberc Lung Dis. 2004;8(10):1234-41.

14. Selvakumar N, Kumar V, Gopi PG, Sivagamasundari S, Prabhakaran E, Vasanthanand S, Narayanan PR. Proficiency to read sputum AFB smears by senior tuberculosis laboratory supervisors under training at a reference laboratory in India. Indian J Tuberc. 2005;52:11-4.

15. Wu MH, Chiang CY, Jou R, Chang SY, Luh KT. External quality assessment of sputum smear microscopy in Taiwan. Int J Tuberc Lung Dis. 2009;13(5):606-12 (PubMed PMID: 19383194).

16. Mosissa Lemi, Kebede Abebaw, Mindaye Tedla, Getahun Muluwork, Tulu Sisay, Desta Kassu. External quality assessment of AFB smear microscopy performances and its associated factors in selected private health facilities in Addis Ababa, Ethiopia. Pan Afr Med J. 2016;24:125. https://doi. org/10.11604/pamj.2016.24.125.7459.

17. Manalebh A, Demissie M, Mekonnen D, Abera B. The quality of sputum smear microscopy in public-private mix directly observed treatment laboratories in West Amhara Region, Ethiopia. PLoS ONE. 2015;10(4):e0123749. https://doi.org/10.1371/journal.pone.0123749.

18. Otero L, Van Deun A, Agapito J, Ugaz R, Prellwitz G, Gotuzzo E, Van der Stuyft P. Quality assessment of smear microscopy by stratified lot sampling of treatment follow-up slides. Int J Tuberc Lung Dis. 2011;15(2):211-6. 
19. Shargie EB, Yassin MA, Lindtjorn B. Quality control of sputum microscopic examinations for acid-fast bacilli in Southern Ethiopia. Ethiop J Health Dev. 2005; 19(2):104-8.

20. Basra D, Matee MIN, McNerney R. Quality assessment of sputum smears microscopy for detection of acid fast bacilli in peripheral health care facilities in Dar es Salaam, Tanzania. East Afr Med J. 2006;83(6):306-10.

21. Mulat M. Quality performance evaluation of laboratories on AFB smears microscopy in Eastern Amhara Region, Ethiopia. Department Of Medical Laboratory Sciences; 2011. Google Scholar.

22. Selvakumar N, Prabhakaran E, Rahman F, Chandu NA, Srinivasan S, Santha $\mathrm{T}$, et al. Blinded rechecking of sputum smears for acid-fast bacilli to ensure the quality and usefulness of restaining smears to assess falsepositive errors. Int J Tuberc Lung Dis. 2003;7(11):1077-82.

23. Ayana DA, Kidanemariam ZT, Tesfaye HM, Milashu FW. External quality assessment for acid fast bacilli smears microscopy in eastern part of Ethiopia. BMC Res Notes. 2015;8:537. https://doi.org/10.1186/s1310 4-015-1478-0

24. Kumar V, Raghavan R, Nagamiah S, Chauhan LS. External quality assessment of smear microscopy by the National Reference Laboratory in nine states of India. Int J Tuberc Lung Dis. 2009:13(9):1183-5.
25. Fujiki A, Giango C, Endo S. Quality control of sputum smear examination in Cebu Province. Int J Tuberc Lung Dis. 2002;6:39-46.

26. Franco C. Calidad del exámen directo de esputo en la Red de Laboratorios de Bacteriología de la TBC de Colombia. Bol Oficina Sanit Panam. 1993;115:103-9.

27. Nyirenda TE, Mundy CJ, Harries AD, Banerjee A, Salaniponi FM. Safety in laboratories carrying out sputum smear microscopy: a dilemma for resource-poor countries. Int J Tuberc Lung Dis. 1998;2(8):690-3 (PubMed PMID: 9712286)

28. Nnaji GA, Chukwu JN. Comparative analysis of errors in reading sputum smears microscopy by supervisors and peripheral laboratory technicians in southeastern Nigeria. Trop J Med Res. 2015;18:80-4.

29. Mycobacteriology Laboratory Manual. World Health Organization Regional Office for the Western Pacific Stop TB, April 2014. http:// www.who.int/tb/laboratory/mycobacteriology-laboratory-manua l.pdf. Accessed 27 June 2017.
Ready to submit your research? Choose BMC and benefit from:

- fast, convenient online submission

- thorough peer review by experienced researchers in your field

- rapid publication on acceptance

- support for research data, including large and complex data types

- gold Open Access which fosters wider collaboration and increased citations

- maximum visibility for your research: over $100 \mathrm{M}$ website views per year

At BMC, research is always in progress.

Learn more biomedcentral.com/submissions 